\title{
Evidence for chemical evolution in the spectra of high redshift galaxies ${ }^{\star}$
}

\author{
D. Mehlert ${ }^{1}$, S. Noll ${ }^{1}$, I. Appenzeller ${ }^{1}$, R. P. Saglia ${ }^{2}$, R. Bender ${ }^{2}$, A. Böhm ${ }^{3}$, N. Drory ${ }^{2}$, K. Fricke ${ }^{3}$, A. Gabasch ${ }^{2}$, \\ J. Heidt ${ }^{1}$, U.Hopp ${ }^{2}$, K. Jäger ${ }^{3}$, C. Möllenhoff ${ }^{1}$, S. Seitz ${ }^{2}$, O. Stahl ${ }^{1}$, and B.Ziegler ${ }^{3}$ \\ 1 Landessternwarte Heidelberg, Königstuhl, 69117 Heidelberg, Germany \\ 2 Universitätssternwarte München, Scheinerstraße 1, 81679 München, Germany \\ ${ }^{3}$ Universitäts-Sternwarte Göttingen, Geismarlandstraße 11, 37083 Göttingen, Germany
}

Received 14 June 2002 / Accepted 1 July 2002

\begin{abstract}
Using a sample of 57 VLT FORS spectra in the redshift range $1.37<z<3.40$ (selected mainly from the FORS Deep Field survey) and a comparison sample with 36 IUE spectra of local $(z \approx 0)$ starburst galaxies we derive C iv and Si iv equivalent width values and estimate metallicities of starburst galaxies as a function of redshift. Assuming that a calibration of the $\mathrm{C}_{\mathrm{IV}}$ equivalent widths in terms of the metallicity based on the local sample of starburst galaxies is applicable to high- $z$ objects, we find a significant increase of the average metallicities from about $0.16 Z_{\odot}$ at the cosmic epoch corresponding to $z \approx 3.2$ to about $0.42 Z_{\odot}$ at $z \approx 2.3$. A significant further increase in metallicity during later epochs cannot be detected in our data. Compared to the local starburst galaxies our high-redshift objects tend to be overluminous for a fixed metallicity. Our observational results are in good agreement with published observational data by other authors and with theoretical predictions of the cosmic chemical evolution.
\end{abstract}

Key words. galaxies: starburst - galaxies: evolution - galaxies: formation - galaxies: stellar content galaxies: fundamental parameters

\section{Introduction}

Among the unsolved questions of cosmology is the beginning and evolution of the star formation process at early cosmic epochs. From the presence of heavy nuclei in high- $z$ quasars and galaxies it is clear that star formation started rather early (see e.g. Hamann \& Ferland 1999; Dietrich et al. 1999). Moreover, galaxy counts and emission line studies of high- $z$ galaxies indicate that the star formation rate (SFR) declined rapidly since at least the epoch corresponding to redshifts of $z \approx 2$ (see e.g. Madau et al. 1996; Madau 2001). On the other hand intrinsic interstellar extinction (which may affect strongly the rest-frame UV of distant galaxies) and uncertainties concerning the relation between line emission and the star formation introduce considerable uncertainties into the derivation of the global star formation rate at early cosmic epochs from visual galaxy counts and emission line studies, while investigations using IR and mm-wave data are hampered by small samples.

An alternative approach to investigate the early star formation history of the universe is the evaluation of the chemical enrichment history of the universe, as star formation and the rapid

Send offprint requests to: D. Mehlert,

e-mail: dmehlert@lsw.uni-heidelberg.de

* Based on observations obtained with the FORS Instruments at the ESO VLT, Paranal, Chile on the course of the observing proposals 65.O-0049(A), 66.A-0547(A), 68.A-0013(A), 68.A-0014(A) evolution of massive stars results in a production of heavy nuclei more or less proportional to the SFR. Steidel et al. (1996a, 1996b) and Lowenthal et al. (1997) have demonstrated that galaxies with redshifts up to about $z \approx 5$ can be observed with optical photometry and spectroscopy during periods of high star formation activity (resulting in high rest-frame UV emission which is redshifted into the optical wavelength range in the observers frame). Basic properties of these objects, such as number densities, luminosities, colors, sizes, morphologies, star formation rates, overall chemical abundances, dynamics and clustering have been investigated in various recent papers (cf. e.g. Steidel et al. 1996b; Yee et al. 1996; Lowenthal et al. 1997; Pettini et al. 2000; Leitherer et al. 2001). Steidel et al. (1996a, 1996b) already noted that the high-z galaxies show, on average, relatively weak metallic absorption lines, and they ascribe this finding tentatively to a lower metal content (a suggestion which has later been reiterated in several subsequent papers). On the other hand most of the published spectra of high- $z$ galaxies are not of sufficient $\mathrm{S} / \mathrm{N}$ to provide qualitative information on the metal content. Therefore, we obtained new high $\mathrm{S} / \mathrm{N}$ spectra of galaxies with $z \leq 3.5$ with the aim of studying the chemical evolution of starburst galaxies at high redshifts.

In the present paper we describe results on the Civ absorption line strength and their interpretation in terms of chemical evolution with cosmic age at redshifts $0<z<3.5$. Most of the new spectra were obtained with the FORS instruments 
at the ESO VLT in the course of a photometric and spectroscopic study of distant galaxies in the FORS Deep Field (FDF) (Appenzeller et al. 2000; Heidt et al. 2001; Bender et al. 2001). Due to its combination of depth and a (compared to the HDFs) relatively large area the FDF is particularly well suited for statistical studies of high-redshift galaxies. In the present investigation we restrict ourselves to redshifts $z \leq 3.5$ since at higher redshifts the position of the redshifted $\mathrm{C}$ IV resonance lines tends to coincide with strong $\mathrm{OH}$ night sky lines. Hence, for an accurate sky subtraction a higher spectral resolution or longer exposure times than we could achieve so far would be needed. In Sect. 2 we describe the sample selection and the observations. In Sect. 3 we present and discuss the measurement of the $\mathrm{C}$ IV (and Si Iv) equivalent widths, from which we estimate the metallicities of the investigated objects in Sect. 4. In Sect. 5 we compare our results with data available in the literature, in Sect. 6 we draw our conclusions.

\section{Sample selection and observations}

The data set used in this study combines spectra of highredshift galaxies observed with the ESO VLT and spectra of local starburst galaxies taken from the IUE archive. 51 of the high-redshift spectra were selected (according to the criteria listed below) from about 300 low-resolution spectra observed mainly during the spectroscopic observing runs of the FDF program (in 3 nights in Sep. and Oct. 2000 and 3.5 nights in Oct. and Dec. 2001). For these observations we used FORS1\&2 at the VLT in MOS and MXU mode with a slit width of $1^{\prime \prime}$ and the 150I grism. (For instrumental details see the FORS Manual at the ESO web page www. eso.org). A few additional spectra had already been obtained during the commissioning phases of FORS1\&2 in 1998, 1999 and 2000 using the same spectroscopical setup as described above. All spectra cover a spectral range (in the observer's frame) from about $3400 \AA$ to about $10000 \AA$ with a spectral scale of $5 \AA /$ pixel and a spectral resolution of about 200. Although spectra of galaxies as faint as $I=26.0 \mathrm{mag}$ have been observed successfully in the FDF, in the present investigation only galaxies with $I \leq 24.5$ mag were included. Depending on the objects' magnitude and the seeing conditions ( 0.7 on average) the integration times ranged between 2 and 12 hours. The data reduction (bias subtraction, flatfielding, cosmic ray elimination, sky subtraction, wavelength calibration, etc.), was performed using standard MIDAS routines. A detailed description of the FDF spectroscopic observing program and the data reduction procedures will be presented by Noll et al. (2002).

With respect to the photometric redshift catalogue of the FDF (see Bender et al. 2001) our spectroscopic sample is complete to about $85 \%$ for our limiting magnitude $(I \leq 24.5 \mathrm{mag}$ ) for photometric redshifts in the range $2.2<z_{\text {phot }}<3.5$. The distribution of the spectroscopic redshifts is in good agreement with the photometric redshift distribution of the FDF which has peaks at redshifts of around 2.4 and 3.4 (see Fig. 3 in Bender et al. 2001). Exceptions are the redshifts in the range $1.4 \leq z \leq 2.2$ where a lack of strong spectroscopic features in our observed wavelength range makes a reliable spectroscopic redshift determination rather difficult, resulting in an artificial low number of objects in our spectroscopic sample.

For the present investigation we selected those FDF galaxies showing absorption line spectra with an adequate $\mathrm{S} / \mathrm{N}$ ( $>10$ per resolution element) for a meaningful quantitative analysis of the $\mathrm{C}$ iv resonance doublet. All these galaxies show typical starburst characteristics in their spectra such as intense (rest frame) UV continua and highly ionized metal absorption lines. Three examples of FDF spectra are displayed in Fig. 1. The 10 high-redshift galaxies which are dominated by their $\mathrm{Ly}_{\alpha}$ emission were not included in our study. This leads to a limitation of the sample to galaxies with $z<4$, since most of the few FDF galaxies with larger redshifts (up to 5.0) observed so far show strong $\operatorname{Ly} \alpha$ emission (while pure absorption line spectra dominate at lower redshifts; see Noll et al. 2002). Also excluded from our study were 4 objects that show absorption lines with clear emission components, forming P-Cygni profiles. (The apparent emission peak redwards from the CIV absorption feature in Fig. 1c is not a P Cygni emission component. The absorption line is unshifted and, as pointed out in the caption, the apparent emission component is an artifact.)

In order to enlarge our sample somewhat we added 6 additional FORS spectra (matching the criteria listed above) which had been observed with the same setup during the FORS commissioning runs (and are now available from the VLT archive). Four of these additional spectra were selected among the gravitationally amplified galaxies behind the cluster 1E0657-558 (Mehlert et al. 2001). We further included two spectra from the HDF-S and AXAF Deep Field follow-up studies (Cristiani et al. 2000), which met our criteria.

The comparison sample of IUE low resolution spectra from the IUE archive $^{1}$ consists of 36 local $(z \approx 0)$ starburst galaxies investigated by Heckman et al. $(1998)^{2}$. All these IUE spectra were obtained with the Short Wavelength Prime Camera in the low dispersion mode and, therefore, cover a similar restframe spectral range with a slightly better spectral resolution as our FORS spectra of high redshift objects. The IUE spectra were reduced using the pipeline provided by the archive and smoothed to attain the same spectral resolution as the VLT spectra.

\section{Equivalent widths}

In an earlier investigation, based on smaller samples of highredshift galaxies, we noticed an apparent anticorrelation between redshift and the strength of the C Iv $1550 \AA$ doublet (Mehlert et al. 2001, 2002). According to Walborn et al. (1995) high-excitation lines like $\mathrm{C}_{\text {IV }}$ and $\mathrm{Si}$ IV are produced mostly in stellar photospheres and winds and their strengths depend sensitively on the stellar metallicity. Although in a few cases a nonnegligible $(\leq 50 \%)$ contamination of the $\mathrm{C}$ IV and Si Iv features

\footnotetext{
http://ines.laeff.esa.es

2 The sample investigated by Heckman et al. (1998) actually contains 45 galaxies. But 3 of these spectra are not available on the IUE archive, and 6 were excluded from our study for having either a too low $\mathrm{S} / \mathrm{N}$ or showing strong emission lines or defects close to the Si IV and/or C Iv lines.
} 

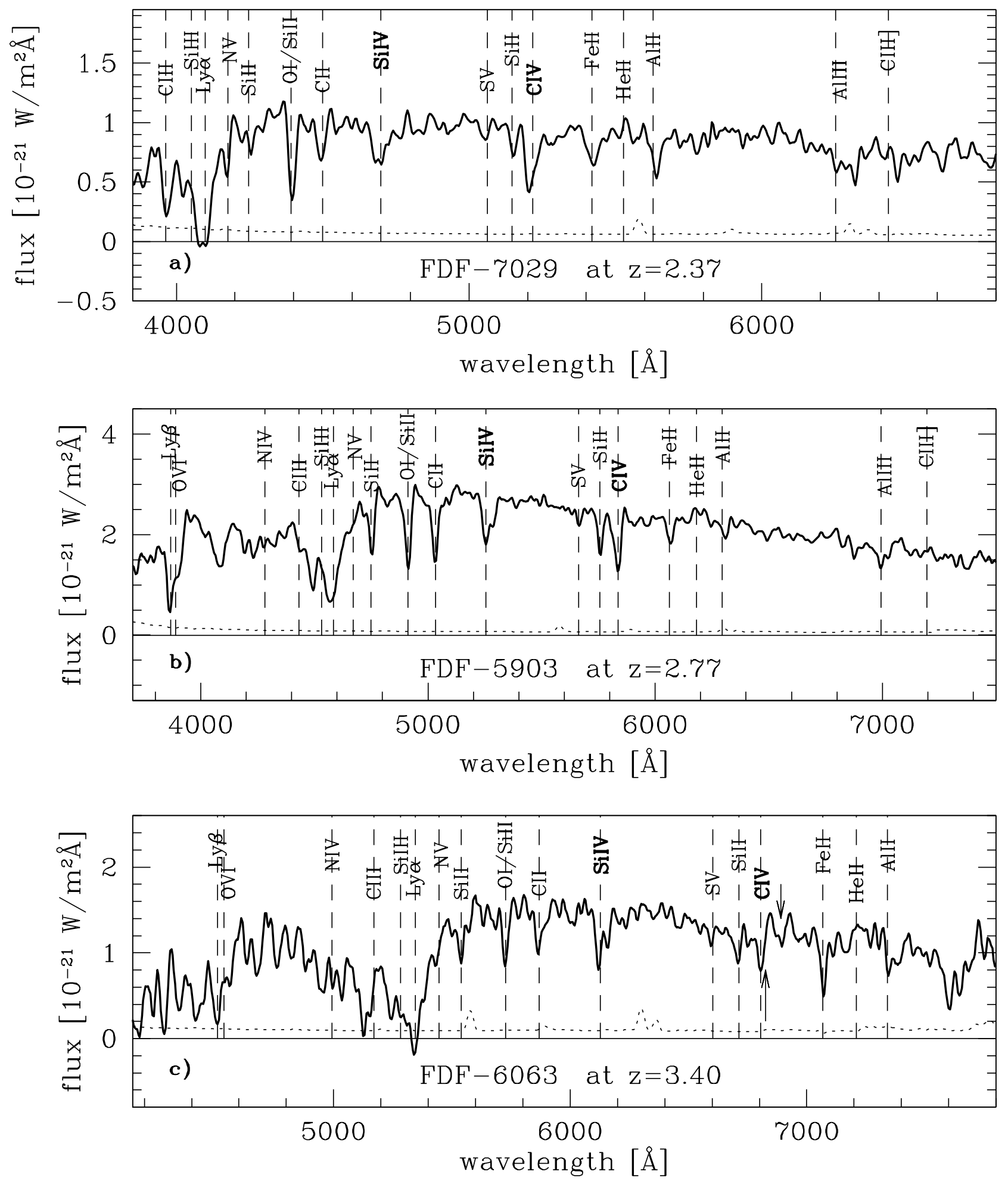

Fig. 1. Examples of the low-resolution galaxy spectra obtained with FORS. The redshift of the objects is increasing from panel a) to c). The dotted line indicates the noise level which (due to the night sky spectrum and the wavelength dependent instrumental efficiency) varies with wavelength. The $\mathrm{S} / \mathrm{N}$ of these spectra is about $\leq 16,43$ and 12 for object 7029, 5903 and 6063, respectively. The object designations correspond to the catalog of Heidt et al. (2002). For orientation the expected positions of selected spectral lines are indicated by vertical dashed lines. The two apparent emission features (marked by arrows) longward of the C IV absorption feature are artifacts and resulted from the increased noise at this wavelengths due to telluric $\mathrm{OH}$ band edges and atmospheric absorption. For the same reason Fig. 1c becomes unreliable beyond $7000 \AA$.

by interstellar absorption could not be excluded, Heckman et al. (1998) find for their sample of 45 nearby starburst galaxies that the C IV and Si Iv absorption is normally produced by photospheric and stellar wind lines of the unresolved stars. 
We found further evidence for a close relation between the strength of these resonance lines and the metallicity of the observed starburst galaxies by measuring the equivalent widths of the C Iv $1550 \AA$ and Si Iv $1398 \AA$ doublets in synthetic spectra of starburst galaxies with different metallicities taken from Leitherer et al. (2001) (see Fig. 2). According to Fig. 2 for ages $\geq 10 \mathrm{Myr}$ the measured equivalent widths depend strongly on the metallicity but are almost independent of the age of the starburst. Therefore, we measured for those galaxies with reliable spectroscopic redshifts $z>1.35$ (i.e. galaxies where the $\mathrm{C}$ IV doublet was redshifted into our observed spectral range) the rest-frame equivalent widths $W_{0}$ of this feature, which is defined by

$W_{0}=\int_{\lambda 1}^{\lambda 2}\left(1-\frac{S(\lambda)}{C(\lambda)}\right) \mathrm{d} \lambda$

Here we used following approximation:

$$
\begin{array}{r}
W_{0}=\Delta \lambda-\frac{1}{C\left(\lambda_{0}\right)} \int_{\lambda 1}^{\lambda 2} S(\lambda) \mathrm{d} \lambda \text { with } \\
\lambda_{1}=\lambda_{0}-\frac{\Delta \lambda}{2} ; \lambda_{2}=\lambda_{0}+\frac{\Delta \lambda}{2} .
\end{array}
$$

The central wavelength for $\mathrm{C}_{\text {IV }}$ is $\lambda_{0}=1549.5 \AA$ and for the width of the line window we chose $\Delta \lambda=30 \AA$. The continuum flux at the central rest frame wavelength $\lambda_{0}$ was approximated by the mean flux within two well defined continuum windows, one on each side of the line window. Each of these continuum windows has a width of $75 \AA$ and is separated from the line window by $5 \AA$. Since the correct continuum determination is critical for the resulting $W_{0}$, its level was checked interactively. In particular it turned out that the influence of the $B$-band, that lies in the right continuum window of the highest $z$ objects is negligible with respect to the resulting $W_{0}$.

For objects with $z>1.7$, where in addition the Si Iv doublet $\left(\lambda_{0}=1398.3 \AA\right)$ became visible, we also measured $W_{0}(\mathrm{Si}$ Iv $)$ with the same bandwidth and continuum window definitions as for $\mathrm{C}$ Iv. The same measurements were also carried out in the IUE spectra of the comparison sample of $z \approx 0$ starburst galaxies. Statistical mean errors of the individual $W_{0}$ measurements were calculated from the $\mathrm{S} / \mathrm{N}$ of the individual spectra and the errors of the continuum fits $(\leq 10 \%)$. Equivalent width measurements in the spectra of faint objects can be affected severely by errors in the sky background subtraction. Therefore, during the reduction of our FORS spectra a particular effort was made to keep these errors low. Various tests showed that for all FORS spectra used in this study the errors in determining the continuum level remained below 5\% (see Noll et al. 2002). Hence, systematic errors in the measured equivalent widths due to an incorrect sky subtraction are well below the statistical errors in most cases.

Another source of systematic errors in our $W_{0}$ measurements is our low spectral resolution, which in most cases did not allow us to resolve the line profiles. However, since equivalent widths measurements of strong isolated lines are in principle independent of the spectral resolution and since all our conclusions are based on differences between measurements carried out with the same procedure in spectra of the same

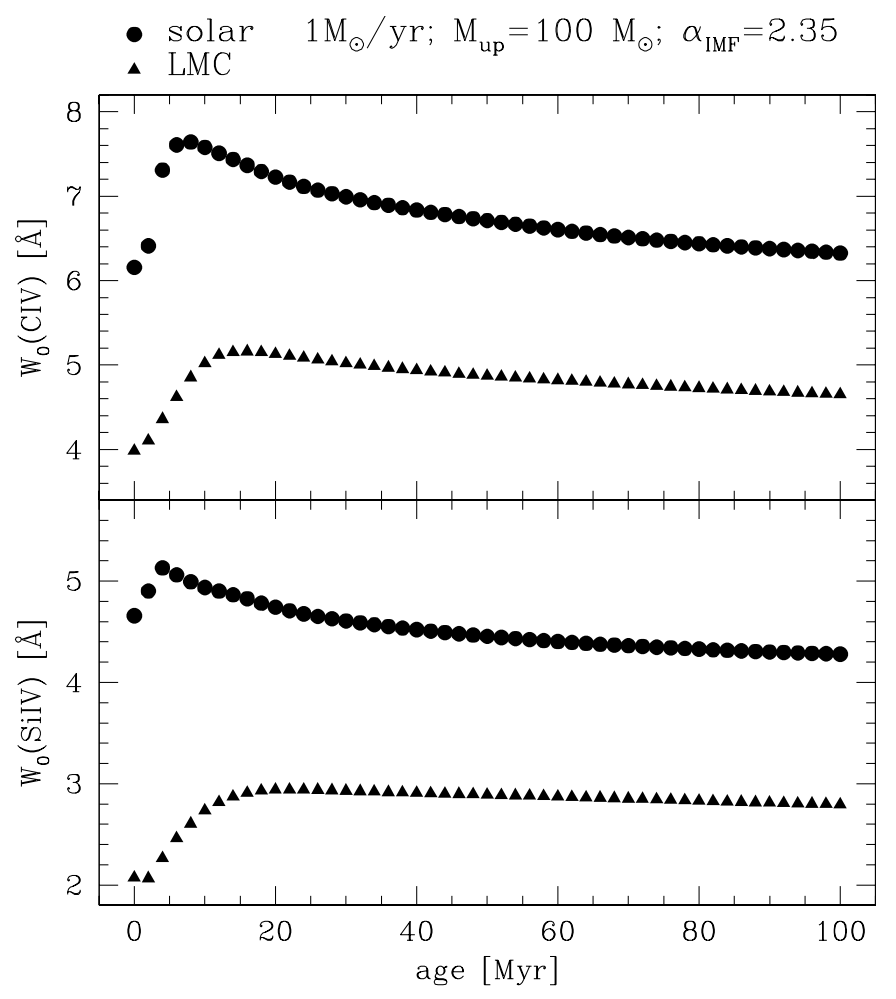

Fig. 2. Measured C Iv (a) and Si Iv (b) equivalent width of the synthetic spectra of Leitherer et al. (2001) as a function of the starburst age. The model spectra are based on continuous star formation $\left(1 M_{\odot} / \mathrm{yr}\right)$ assuming the model parameter $M_{\mathrm{up}}=100 M_{\odot}$ and $\alpha_{\mathrm{IMF}}=2.35$. Circles and triangles correspond to solar and LMC metallicity, respectively.

resolution, these systematic errors are expected to cancel out and therefore are not expected to affect the results of this paper significantly. On the other hand, since the $\mathrm{C}_{\mathrm{IV}}$ doublet is not a truly isolated feature, our numerical results for the equivalent widths should not be directly compared to results obtained from spectra with a different spectral resolution.

The results of our $W_{0}\left(\mathrm{C}_{\text {IV }}\right)$ measurements are listed in Table 1 and plotted in Fig. 3. In order to avoid a crowding of data points at $z \approx 0$, we plotted for the local (IUE) starburst galaxies only the average value and indicate the $1 \sigma$ scatter of the individual values by a bar. For the high-redshift galaxies the individual data points and their mean errors are given.

As demonstrated by Fig. 3 our high-redshift galaxies with $z<2.5$ show about the same average $\mathrm{C}_{\text {Iv }}$ equivalent widths and about the same scatter around the average as the local starburst galaxies. However, for redshifts larger than about 2.5 the average $\mathrm{C}$ Iv equivalent widths and their scatter clearly decrease with $z$ in our sample. As described in Sect. 2 this decrease is not driven by any selection effect since the spectroscopic redshift distribution of the included FDF galaxies is in good agreement with the photometric redshift distribution.

Figure 3 obviously confirms the effect suspected by Steidel et al. (1996a) quantitatively. In order to estimate (in view of the observed scatter) the statistical significance of the effect, we calculated averages and their mean errors of the $W_{0}(\mathrm{C}$ IV $)$ values for selected redshift bins. The results are listed in Table 2 and plotted in Fig. 4. The table confirms that there is no 
Table 1. Measured $\mathrm{C}_{\mathrm{IV}}$ and Si IV rest-frame equivalent widths for the 57 high- $z$ galaxies. The absolute $B$-magnitudes derived and discussed in Sect. 5 are also listed.

\begin{tabular}{|c|c|c|c|c|c|c|c|}
\hline No. & $z$ & $\begin{array}{c}I \\
{[\mathrm{mag}]}\end{array}$ & $\begin{array}{c}W_{0}\left(\mathrm{C}_{\text {IV }}\right) \\
{[\AA]}\end{array}$ & $\begin{array}{c}\mathrm{d} W_{0}(\mathrm{C} \text { IV }) \\
{[\AA]}\end{array}$ & $\begin{array}{c}W_{0}(\mathrm{Si} \text { IV }) \\
{[\AA]}\end{array}$ & $\begin{array}{c}\mathrm{d} W_{0}(\mathrm{Si} \text { IV }) \\
{[\AA]}\end{array}$ & $\begin{array}{c}M_{B} \\
{[\mathrm{mag}]}\end{array}$ \\
\hline FDF-1208 & 2.18 & 23.68 & 3.92 & 0.41 & 3.31 & 0.44 & -22.45 \\
\hline FDF-1331 & 3.39 & 23.89 & 1.80 & 0.90 & - & - & -23.30 \\
\hline FDF-1555 & 3.26 & 23.88 & 1.16 & 0.74 & 1.29 & 0.69 & -22.36 \\
\hline FDF-1578 & 2.71 & 24.25 & 3.545 & 0.90 & 2.97 & 0.81 & -21.98 \\
\hline FDF-1691 & 2.34 & 23.89 & 3.65 & 0.57 & 4.30 & 0.60 & -21.53 \\
\hline FDF-1709 & 1.67 & 24.33 & 6.07 & 0.75 & - & - & -20.46 \\
\hline FDF-1744 & 2.37 & 24.10 & 2.34 & 0.58 & 4.27 & 0.56 & -22.21 \\
\hline FDF-1922 & 1.83 & 23.36 & 3.62 & 0.28 & 0.95 & 0.38 & -21.64 \\
\hline FDF-2033 & 2.75 & 24.08 & 5.51 & 0.65 & 4.05 & 0.52 & -21.62 \\
\hline FDF-2274 & 2.25 & 23.34 & 1.62 & 0.31 & 2.66 & 0.33 & -21.66 \\
\hline FDF-2418 & 2.33 & 23.16 & 6.57 & 0.47 & 6.36 & 0.46 & -23.19 \\
\hline FDF-2495 & 2.45 & 23.31 & 3.98 & 0.32 & 3.24 & 0.32 & -22.32 \\
\hline FDF-2636 & 2.25 & 23.43 & 5.21 & 0.63 & 6.41 & 0.70 & -22.77 \\
\hline FDF-3005 & 2.25 & 23.51 & 6.95 & 0.38 & 8.18 & 0.43 & -22.63 \\
\hline FDF-3163 & 2.44 & 23.35 & 4.80 & 0.33 & 5.45 & 0.33 & -22.97 \\
\hline FDF-3173 & 3.27 & 23.91 & 2.59 & 0.48 & 4.24 & 0.44 & -22.51 \\
\hline FDF-3300 & 2.37 & 23.91 & 2.14 & 0.42 & 2.16 & 0.41 & -21.79 \\
\hline FDF-3374 & 2.38 & 23.34 & 5.05 & 0.30 & 5.21 & 0.27 & -22.65 \\
\hline FDF-3810 & 2.37 & 22.67 & 4.95 & 0.25 & 5.59 & 0.26 & -23.18 \\
\hline FDF-3874 & 2.48 & 23.30 & 2.66 & 0.43 & 3.83 & 0.43 & -23.15 \\
\hline FDF-3875 & 2.24 & 24.53 & 4.19 & 0.51 & 3.38 & 0.52 & -20.73 \\
\hline FDF-3958 & 2.13 & 23.87 & 4.40 & 0.53 & 1.43 & 0.57 & -20.98 \\
\hline FDF-3999 & 3.39 & 24.00 & 3.74 & 0.65 & 7.89 & 0.56 & -22.70 \\
\hline FDF-4049 & 1.48 & 23.00 & 4.42 & 0.53 & - & - & -21.76 \\
\hline FDF-4795 & 2.16 & 23.31 & 5.80 & 0.36 & 5.93 & 0.39 & -22.35 \\
\hline FDF-4871 & 2.47 & 23.39 & 7.14 & 0.35 & 6.05 & 0.34 & -22.62 \\
\hline FDF-4996 & 2.03 & 23.25 & 3.35 & 0.37 & 1.19 & 0.44 & -21.77 \\
\hline FDF-5058 & 2.03 & 23.34 & 4.82 & 0.25 & 3.40 & 0.27 & -21.57 \\
\hline FDF-5072 & 1.39 & 22.45 & 3.27 & 0.57 & - & - & -21.88 \\
\hline FDF-5135 & 2.34 & 23.62 & 2.31 & 0.71 & 1.99 & 0.77 & -22.73 \\
\hline FDF-5152 & 1.37 & 22.65 & 3.91 & 0.50 & - & - & -21.55 \\
\hline FDF-5165 & 2.35 & 23.26 & 5.73 & 0.55 & 6.02 & 0.53 & -23.02 \\
\hline FDF-5190 & 2.35 & 24.39 & 2.73 & 0.68 & 2.65 & 0.64 & -22.74 \\
\hline FDF-5215 & 3.15 & 22.98 & 2.54 & 0.47 & 2.45 & 0.40 & -23.18 \\
\hline FDF-5227 & 2.40 & 23.85 & 4.73 & 0.72 & 2.02 & 0.79 & -21.79 \\
\hline FDF-5504 & 3.38 & 23.63 & 3.29 & 1.00 & 7.19 & 0.73 & -23.65 \\
\hline FDF-5550 & 3.38 & 23.12 & 2.44 & 0.41 & 5.23 & 0.31 & -23.23 \\
\hline FDF-5903 & 2.77 & 22.33 & 4.02 & 0.21 & 4.32 & 0.16 & -23.23 \\
\hline FDF-6024 & 2.37 & 22.00 & 4.93 & 0.20 & 5.71 & 0.19 & -23.34 \\
\hline FDF-6063 & 3.40 & 22.56 & 1.36 & 0.49 & 4.24 & 0.42 & -23.35 \\
\hline FDF-6069 & 2.68 & 24.22 & 5.36 & 0.81 & 3.45 & 0.70 & -21.74 \\
\hline FDF-6287 & 2.68 & 24.11 & 1.61 & 0.82 & - & - & -22.04 \\
\hline FDF-6372 & 2.35 & 23.38 & 3.20 & 0.37 & 3.43 & 0.35 & -22.18 \\
\hline FDF-6407 & 2.16 & 23.59 & 5.29 & 0.59 & 4.26 & 0.72 & -22.31 \\
\hline FDF-6864 & 1.39 & 23.41 & 4.93 & 0.71 & - & - & -20.87 \\
\hline FDF-6934 & 2.44 & 22.90 & 4.71 & 0.56 & 3.01 & 0.60 & -23.05 \\
\hline FDF-6947 & 2.36 & 23.83 & 5.91 & 0.42 & 4.90 & 0.45 & -21.94 \\
\hline FDF-7029 & 2.37 & 23.63 & 6.88 & 0.42 & 5.82 & 0.42 & -23.07 \\
\hline FDF-7307 & 2.44 & 24.07 & 2.51 & 0.60 & 3.05 & 0.62 & -21.20 \\
\hline FDF-7342 & 2.37 & 23.80 & 6.13 & 0.76 & 4.58 & 0.75 & -22.06 \\
\hline FDF-7539 & 3.29 & 23.51 & 1.92 & 0.46 & 4.22 & 0.41 & -22.41 \\
\hline ES0657-A & 2.34 & 24.50 & 5.88 & 0.48 & 3.98 & 0.45 & - \\
\hline ES0657-C & 3.08 & 24.89 & 2.30 & 1.00 & 7.07 & 0.62 & - \\
\hline ES0657-J & 2.61 & 22.98 & 3.81 & 0.42 & 2.41 & 0.26 & - \\
\hline ES0657-Core & 3.24 & 24.31 & 2.01 & 0.63 & 3.45 & 0.53 & - \\
\hline HDFS-047 & 2.79 & - & 2.50 & 0.30 & 4.40 & 0.17 & - \\
\hline AXAF-028 & 3.13 & - & 2.84 & 1.00 & 3.45 & 0.97 & - \\
\hline
\end{tabular}




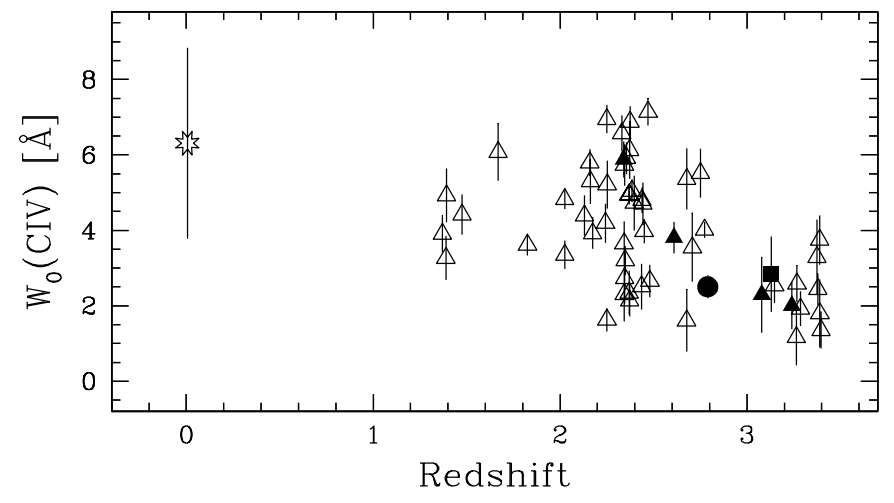

Fig. 3. Measured C IV $\lambda 1550$ rest-frame equivalent widths as a function of redshift. The various symbols have the following meaning: Open star at $z \approx 0$ : Average and $1 \sigma \mathrm{rms}$ scatter for the 36 (Heckman et al. 1998) local starburst galaxies. Open triangles: FDF galaxies. Filled triangles: Galaxies in the field of the cluster 1E0657-558 (Mehlert et al. 2001). Filled circle and square: Galaxies in the HDF-S and the AXAF Deep Field, respectively (Cristiani et al. 2000).

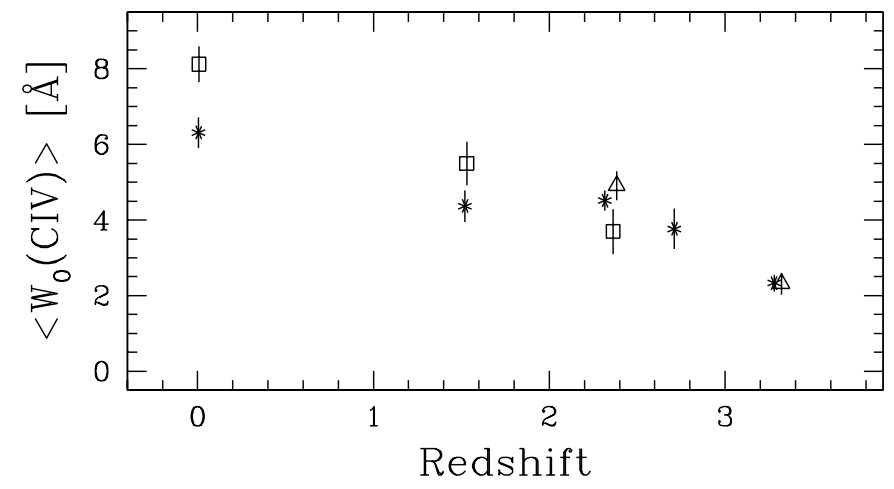

Fig. 4. Averages of the measured $C_{\text {IV }} \lambda 1550$ rest frame equivalent widths within selected redshift bins (see Table 2) as a function of redshift for all galaxies shown in Fig. 3 (asterisks). The bars indicate the mean errors of the averages. Open triangles show all FDF galaxies brighter than $M_{B}=-22.28 \mathrm{mag}$, open squares all FDF galaxies with $-21.52 \mathrm{mag} \leq M_{B} \leq-20.38 \mathrm{mag}$ (see Sect. 5 for discussion).

statistically significant difference between the results for the first three bins while the difference between the local sample and our starburst galaxies with $z>3.0$ is highly significant $(>9 \sigma)$. Tests with other bin sizes and binning intervals showed that the high significance of the result persists for any reasonable bin distribution.

In Fig. 5 we present analogously to Fig. 3 the observed Si IV equivalent width values as a function of redshift. Similarly as in the case of the $\mathrm{C}$ IV doublet the average Si IV strength does not change with redshift for $z<2.5$. However, unlike the average C Iv strength the average Si Iv $W_{0}$ values remain at the local value even beyond $z>2.5$. As a result, the ratio between the Si IV and C IV resonance doublets, which is practically constant for low $z$, varies for high redshifts in our sample. This is demonstrated quantitatively by Table 3 and Fig. 6 .

As noted above, for local starburst galaxies the C IV doublet as well as the Si Iv doublet are good metallicity indicators. Since both elements are produced during the evolution and explosion of massive stars, a greatly different relative chemical
Table 2. Averages and mean errors of the measured $\mathrm{C}$ iv $\lambda 1550$ rest frame equivalent widths within selected redshift bins for all galaxies listed in Table 1. $N$ is the number of objects within each bin.

\begin{tabular}{lcccr}
\hline \hline$z$ interval & $\langle z\rangle$ & $\begin{array}{c}\left.<\mathrm{C}_{\text {IV }}\right\rangle \\
{[\AA]}\end{array}$ & $\begin{array}{c}\text { m.e.(C IV) } \\
{[\AA]}\end{array}$ & $N$ \\
\hline $0.00-0.02$ & 0.01 & 6.31 & 0.41 & 36 \\
$1.00-1.99$ & 1.52 & 4.37 & 0.41 & 6 \\
$2.00-2.49$ & 2.32 & 4.51 & 0.27 & 32 \\
$2.50-2.99$ & 2.71 & 3.77 & 0.53 & 7 \\
$\geq 3.00$ & 3.28 & 2.33 & 0.22 & 12 \\
\hline
\end{tabular}

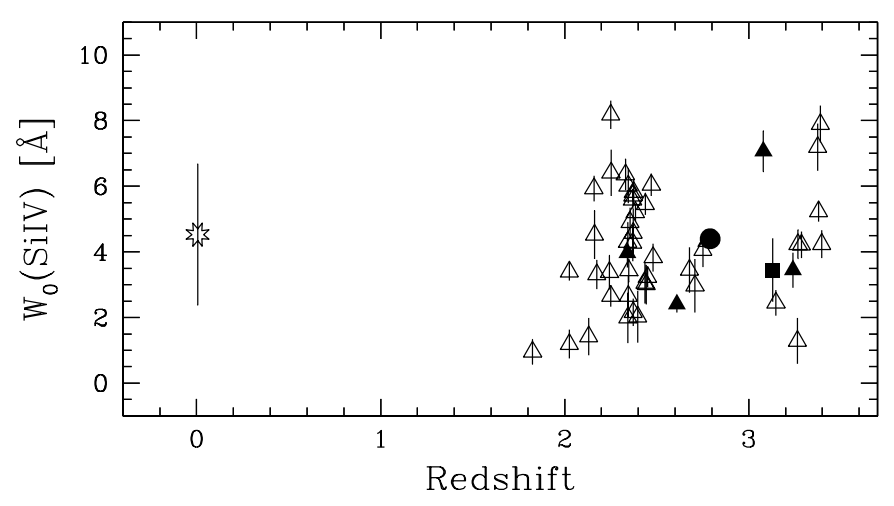

Fig. 5. Measured Si Iv $\lambda 1400$ rest-frame equivalent width as a function of redshift. Symbols as is Fig. 3.

abundance of $\mathrm{C}$ and $\mathrm{Si}$ in the high- $z$ starburst galaxies appears very unlikely. In spite of the unsatisfactory state of present SN II models and the remaining large uncertainties concerning the intermediate mass element yields for different initial stellar masses, it seems very difficult to enhance Si production relative to $\mathrm{C}$. On the other hand, it is well known that individual hot stars of the same metallicity show a wide range of $\mathrm{C}$ IV to $\mathrm{Si}$ Iv line ratios. Strong $\mathrm{C}$ IV absorption is well known to be universally present in $\mathrm{O}$ stars of all luminosity classes, while $W_{0}$ (Si Iv) is luminosity dependent and decreases rapidly from supergiant stars to dwarfs (Walborn \& Panek 1984; Pauldrach et al. 1990; Leitherer et al. 1995). This line is, therefore, used as a luminosity indicator in UV stellar classification schemes. Moreover, Si Iv has a pronounced maximum in early B stars while $\mathrm{C}$ iv changes monotonically with temperature. Finally the Si IV strength is more strongly affected by population differences (i.e. stellar age differences) than the C IV doublet. A scatter in these population differences can easily mask any metallicity dependence in the Si IV line strength.

Hence, assuming that the observed absorption lines are dominated by contributions of the stellar photospheres and winds, the $W_{0}(\mathrm{Si}$ IV $) / W_{0}(\mathrm{C}$ IV $)$ ratio can, in principle, be used to derive informations on the star formation history (instantaneous or continuous) and/or the stellar mass distribution (see also Mas-Hesse \& Kunth 1991). Therefore, Fig. 6 can possibly be understood by assuming that at the epochs corresponding to $z>3$ (i.e. during the first two Gyrs of the universe) instantaneous star bursts played an important role while "continuous star formation" is the normal mode for local and lower 


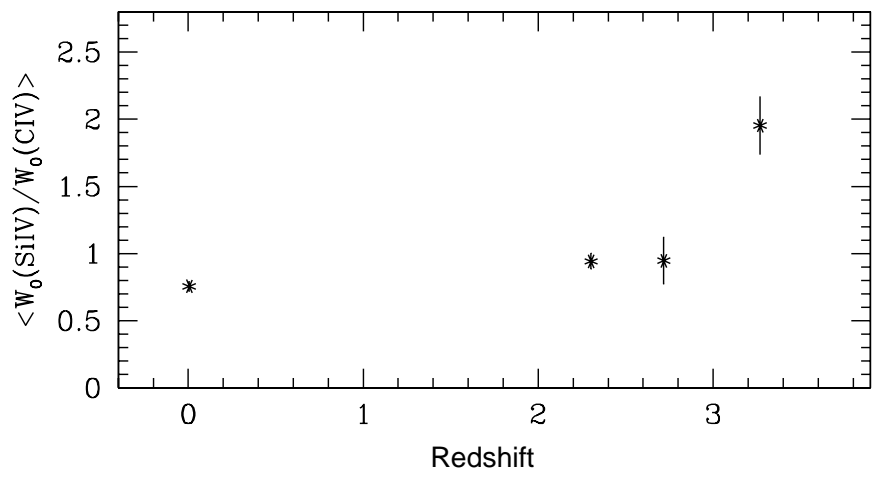

Fig. 6. Averages of the ratios between the measured Si IV and $\mathrm{C}_{\text {IV }}$ rest frame equivalent widths as a function of redshift for all galaxies shown in Fig. 5. The mean error of each average, calculated from the scatter of the individual values, is indicated by a bar.

Table 3. Average and mean error of the ratios between the measured $\mathrm{Si}$ iv and $\mathrm{C}$ iv rest frame equivalent widths within selected redshift bins for all high- $z$ galaxies for which $W_{0}(\mathrm{Si}$ Iv $)$ has been measured. $N$ is the number of galaxies within each bin.

\begin{tabular}{cccc}
\hline \hline$<z>$ & $\langle$ Si IV/C IV $>$ & m.e.(Si IV/C IV) & $N$ \\
\hline 0.00 & 0.76 & 0.04 & 36 \\
2.30 & 0.94 & 0.06 & 33 \\
2.72 & 0.95 & 0.18 & 6 \\
3.27 & 1.95 & 0.22 & 11 \\
\hline
\end{tabular}

redshift starburst galaxies. However, since the different parameters which determine the value of $W_{0}(\mathrm{Si}$ Iv $) / W_{0}(\mathrm{C}$ IV $)$ cannot be disentangled reliably without including additional lines in the analysis, spectra of higher resolution and higher $\mathrm{S} / \mathrm{N}$ are required to finally settle this issue.

The investigation of the purely interstellar lines of lower ionisation like e.g. Si II $\lambda$ 1260, O I/Si II $\lambda 1303$ and C II $\lambda 1335$ is in progress and the results will be published in a separate paper.

\section{Metallicities}

Since, in contrast to the variations of the $\mathrm{Si}$ Iv line, differences of the $C_{\text {IV }}$ line strength cannot be easily explained by population differences in the starburst galaxies, the observed decrease of the $\mathrm{C}$ IV equivalent width values for $z>2.5$ in our sample can at present only be interpreted as a metallicity effect. Hence, the decrease of $W_{0}(\mathrm{C}$ IV $)$ with $z$ is expected to contain information on the evolution of the metal content of starburst galaxies with cosmic age. In order to derive a more quantitative measure of the metallicity evolution apparently observed in Fig. 3, we made an attempt to calibrate the observed $W_{0}(\mathrm{C}$ IV $)$ values in terms of the $\mathrm{O} / \mathrm{H}$ ratios. For this purpose we used the oxygen abundances listed in Heckman et al. (1998) for all the local starburst galaxies of this sample and derived metallicities using the relation $\log Z=12+\log (\mathrm{O} / \mathrm{H})$.

In Fig. 7 we plotted for the local starburst galaxies the metallicity relative to the solar value $\left(\log Z_{\odot}=8.93\right)$ as a function of our measured $W_{0}(\mathrm{C}$ IV $)$ values. We also included the

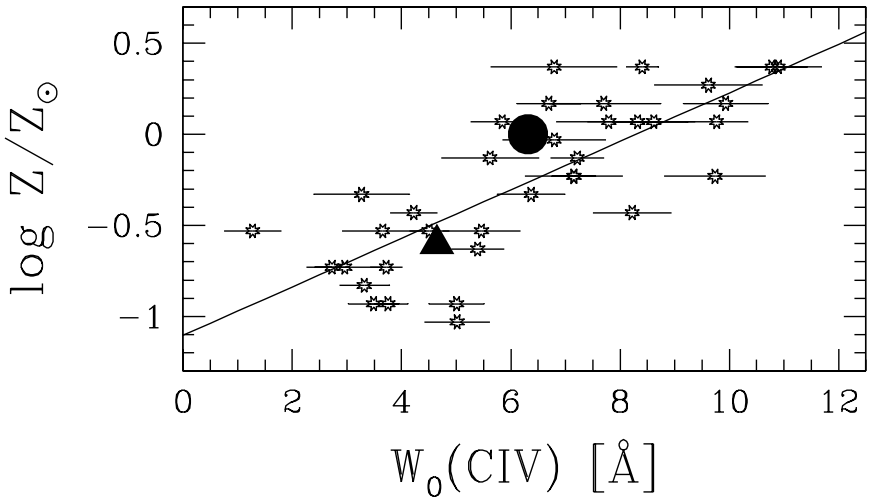

Fig. 7. Metallicity $\log Z / Z_{\odot}$ in terms of oxygen abundance of 36 local starburst galaxies (from Heckman et al. 1998) as a function of the measured $C_{\text {Iv }} \lambda 1550$ equivalent width (open stars). The typical error for $\log Z / Z_{\odot}$ is $\leq 0.1$ dex. The solid line gives the best linear fit to the data. For comparison the $W_{0}(\mathrm{C}$ iv $)$ of synthetic starburst spectra (from Leitherer et al. 2001; see Fig. 2) at an age of $100 \mathrm{Myr}$ for solar (filled circle) and LMC ( 0.25 solar, filled triangle) metallicity have also been included in the figure.

theoretical values determined for synthetic starburst galaxies with solar and LMC metallicities taken from Leitherer et al. (2001). Although the scatter is rather large $(\mathrm{rms}=0.27)$, the plot indicates a dependence of $\log \mathrm{Z}$ on $W_{0}(\mathrm{C}$ IV $)$ which can be approximated by a linear relation. The best linear least square fit to these data gives

$\log Z / Z_{\odot}=0.13( \pm 0.02) \times W_{0}(\mathrm{C}$ IV $)-1.10( \pm 0.12)$.

This calibration of the C IV strength in terms of metallicity should be a reasonable approximation for statistical applications at least for local starburst galaxies and the objects with $z<2.5$. However, in view of the population differences evident from the different $\mathrm{Si}$ IV to $\mathrm{C}$ IV ratio, its applicability to the $z>2.5$ objects is less clear. Nevertheless, because of the absence of other more reliable calibration procedures, and since (in view of the physics of hot stars) the relation between the $C_{\text {IV }}$ strength and the metallicity should not be much affected by population details of starbursts we will assume for the following that the correlation between the oxygen abundances and $W(\mathrm{C}$ IV $)$ observed for low redshift starburst galaxies is also valid at high redshifts. With this assumption we convert our observed $\mathrm{C}$ IV equivalent width values to metallicities using Eq. (3). In this way we obtain for our starburst galaxies with $z>$ $3(\langle z\rangle=3.24)$ an average metallicity of about $0.16 Z_{\odot}$ and for $\langle z\rangle=2.34$ a value of $0.42 Z_{\odot}$. The corresponding local $(z=$ 0 ) value would be $0.56 Z_{\odot}$. In terms of cosmic time scales (for a universe with $\Omega_{\Lambda}=0.7, \Omega_{M}=0.3, H_{0}=67 \mathrm{~km} \mathrm{~s}^{-1} \mathrm{Mpc}^{-1}$ used throughout the paper) this would correspond to an increase of the mean metallicity in starburst galaxies by a factor of 2.5 within $\approx 1$ Gyr between cosmic ages of about 1.9 Gyrs and 2.9 Gyrs. For later epochs the data suggest only little further enrichment. Because of the approximative nature of Eq. (3) these numbers are rough estimates only. Still, they agree surprisingly well with earlier theoretical predictions of the cosmic chemical enrichment history of the universe by e.g. Fritze-von Alvensleben (1998) and by Renzini (1998, 2000), 
who predicts that the metallicity had been $\approx 0.1 Z_{\odot}$ at $z=3$ and has increased to a value of $\approx 1 / 3 Z_{\odot}$ in the local universe.

Two effects may affect the validity of Eq. (3) at high redshifts: First, in the IUE spectra of the local starburst galaxies contributions of the Milky Way halo components are present, while they are absent in the high- $z$ spectra. Savage \& Massa (1987) showed that the Civ equivalent width of distant halo stars are normally $<0.5 \AA$. This is one order of magnitude lower than the values measured in the local starburst galaxies. Hence its effect on Eq. (3) should be on a 10\% level, at most. Secondly, the spectrum of the magnified high- $z$ object MS1512-cb58 $(z=$ 2.727) published by Pettini et al. (2000) suggests that the contribution of the interstellar line to the $\mathrm{C}_{\mathrm{IV}}$ absorption feature increases with redshift. If this holds for all galaxies at similar redshifts, this could result in a general difference of the mean $\mathrm{C}$ IV equivalent width between local starburst galaxies and highredshift objects, but it could not explain the observed evolution of the C IV equivalent width between $z=2.5$ and $z=3.5$. Furthermore, by applying the local relation to high redshifts we would overestimate the mean metallicity at young epochs.

\section{Luminosity effects}

When interpreting the data described above one has to keep in mind that for local $(z \approx 0)$ galaxies the metallicities are known to depend on the galaxies' blue and infrared luminosities, with luminous galaxies tending to have higher metallicities (see e.g. Kobulnicky \& Zaritsky 1999; Heckman et al. 1998). Since at high redshifts we observe only very luminous galaxies. Therefore, if a metallicity-luminosity correlation exists these bright objects should be metal rich and we should find an opposite correlation between metallicity and redshift than the one detected in this work. To test whether at high redshifts a metallicity-luminosity correlation does exist and may affect our detected metallicity evolution we plotted in Fig. 8 the absolute $B$-magnitudes $M_{B}$ of all high-redshift FDF galaxies as well as $M_{B}$ for the local starburst galaxies. For the local objects the $M_{B}$ was taken from Heckman et al. 1998 (transformed to the cosmology used in this paper), for the FDF galaxies we computed $M_{B}$ as follows: We derived the best fitting SED, scaled to the total $I$ flux derived by SExtractor (FLUX_AUTO) as determined by our photometric redshift code (see Bender et al. 2001). Then this SED was transformed to $z=0$ (using the observed spectroscopic redshift) to derive the rest-frame $B$-magnitude of the galaxies. Since for the redshift range in question the measured $J$ and $K$ bands bracket the rest-frame $B$, this procedure is nearly equivalent to an interpolation, minimizing the uncertainties in the $\mathrm{K}$ corrections. A detailed description of the method can be found in Gabasch et al. (2002). Using the photometric instead of the spectroscopic redshifts would produce a typical variations of $\pm 0.2 \mathrm{mag}$. Absolute magnitudes were derived assuming the cosmology parameter $H_{0}=67, \Omega_{m}=0.3, \Omega_{\Lambda}=0.7$. Our $M_{B}$ have been corrected for foreground Galactic extinction but not for any internal extinction in the starburst galaxies. From Fig. 8 we see that the local starburst galaxies indeed show the expected correlation between $W_{0}(\mathrm{C}$ IV $)$ and the luminosity. On the other hand, for the high-redshift galaxies we cannot determine whether a metallicity-luminosity relation does exist or

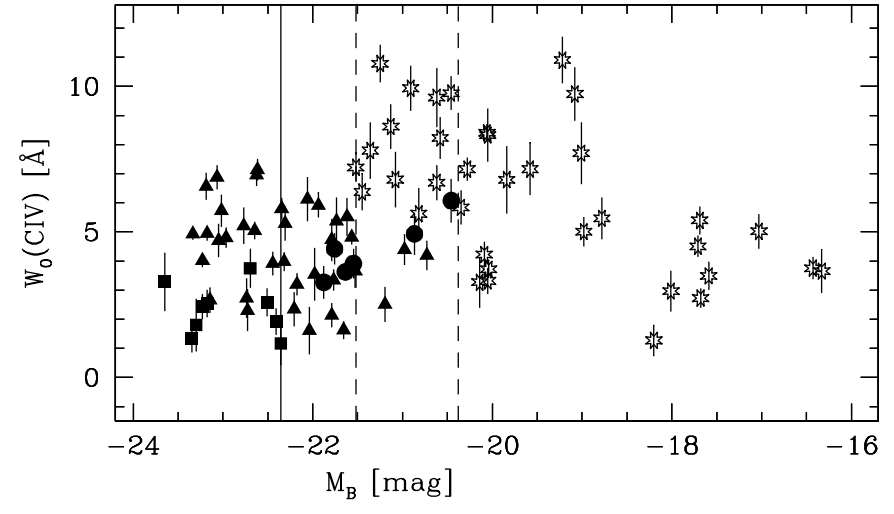

Fig. 8. Measured $C_{\text {IV }} \lambda 1550$ rest-frame equivalent widths of the local starburst galaxies (open stars) and the high- $z$ FDF (filled symbols) versus the absolute $B$-magnitude. For the latter ones we indicated objects within the different redshift bins $1 \leq z<2,2 \leq z<3$ and $z \geq 3$ by filled circles, triangles and squares, respectively. The solid vertical line indicates $M_{B}=-22.36 \mathrm{mag}$, while the dashed vertical lines indicate $M_{B}=-21.52 \mathrm{mag}$ and $M_{B}=-20.38 \mathrm{mag}$.

not, since we do not have any faint objects in our high- $z$ sample. But it is evident that the high-redshift galaxies are on average overluminous for their metallicities compared with local starburst galaxies. This agrees well with earlier results from Pettini et al. (2001) and Kobulnicky \& Koo (2000) who find this trend for Lyman-break galaxies. Hence, if a metallicity-luminosity relation does exist at high redshifts, our data suggest that it has a clear offset to the local correlation, which seems to evolve with redshift. Moreover, from Fig. 8 it is obvious that for the high-redshift galaxies there is no correlation between the measured $W_{0}\left(\mathrm{C}_{\mathrm{IV}}\right)$ and the luminosity that could cause the correlation with $z$ found in this paper.

Since at high redshifts we do not have any faint objects in our sample, while in the local universe we do not find bright starburst galaxies, we have to make sure that our detected metallicity evolution with redshift is not produced by comparing different objects at different redshifts. For that reason we separately investigated all galaxies, which are brighter than the faintest one at $z \geq 3$ (which is $M_{B}=-22.36 \mathrm{mag}$; solid line in Fig. 8). In our sample we only find galaxies brighter than this limit for $z \geq 2$. Their average values of the measured $W_{0}(\mathrm{C}$ IV $)$ and the mean error at redshift 2.4 and 3.3 as well as the single $W_{0}(\mathrm{C}$ IV $)$ measurement for this brightest local galaxy are additionally indicated by open triangles in Fig. 4 . Obviously these subsample show the same trend with decreasing redshift as the total galaxy sample at $z \geq 2$. Furthermore we investigated all galaxies fainter than $M_{B}=-21.52 \mathrm{mag}$ (brightest local galaxy) and brighter than $M_{B}=-20.38 \mathrm{mag}$ (faintest galaxy with $z \geq 1$ ). The average values of the measured $W_{0}(\mathrm{C}$ Iv $)$ and the mean error at redshift $0,1.5$ and 2.4 are also indicated in Fig. 4 by open squares and again show the same trend with decreasing redshift. From these test we conclude that the observed dependence of $W_{0}(\mathrm{C}$ IV $)$ on redshift is not caused by a luminosity effect. Moreover the two open symbols in Fig. 4 for $z \approx 2.4$ indicate that a metallicity-luminosity correlation also exists at this redshift. 
The following additional selection effects could be present (and possibly weaken) the observed correlation between metallicity and redshift at high-z: It could, in principle, be possible that at high- $z$ we preferentially see objects with low internal extinction, which have low dust content and hence low metallicity. In this case we would expect to find a negative correlation between the UV luminosity of our galaxies and their metallicity. To test whether this correlation is present in our high- $z$ galaxies we calculated the UV luminosity as follows:

$$
\begin{aligned}
\log \left(L_{\mathrm{UV}}\right)=\log <F & >{ }_{1432}^{1532}+\frac{(m-M)}{2.5} \\
& +\log \left(4 \pi(10 p c)^{2}\right)
\end{aligned}
$$

where $\langle F\rangle_{1432}^{1532}$ is the mean flux between $1432 \AA$ and $1532 \AA$ (as defined by Kinney et al. 1993), $(m-M)$ is the distance modulus calculated using the cosmological parameters mentioned above. We did not correct for intrinsic reddening since such a correction would have involved considerable uncertainties and is not needed for the the present test. From Fig. 9, where we plotted the $\mathrm{C}$ IV equivalent widths for the high- $z$ FDF galaxies as a function of $L_{\mathrm{UV}}$, we see that no such correlation is evident for our high- $z$ objects. Hence our galaxy sample seems not to be affected by this selection effect.

\section{Comparison with literature data}

Although most published spectra of high redshift galaxies lack the $\mathrm{S} / \mathrm{N}$ required to carry out a study of the type presented here we tried to compare our results with the limited information available on this subject in the literature. For this purpose we measured the $\mathrm{C}$ IV equivalent width for all high- $z$ galaxy spectra published in the papers listed in the caption of Table 4. As in our samples, spectra with strong $\operatorname{Ly} \alpha$ emission were disregarded, and only equivalent width values with estimated (rest frame) mean errors $\leq 1.0 \AA$ were used for the comparison. The number of the (for our purpose) usable spectra of each publication is listed in Col. 4 of Table 4. (Tests showed that including less accurate data lead to similar results, but with much larger statistical errors). For the objects observed by Yee et al. (1996) and by Pettini et al. $(1998,2000)$ the author kindly made their spectra available to us in electronic form. Hence, to measure $W_{0}(\mathrm{C}$ Iv $)$ in these literature objects we were able to apply exactly the same procedure as used for the galaxies presented in this work. For the objects investigated by Steidel et al. (1996a, 1998), Lowenthal et al. (1997) and Trager et al. (1997) we measured the $W_{0}(\mathrm{C}$ iv $)$ from enlarged tracings. We tested the reliability of measuring $W_{0}(\mathrm{C}$ IV $)$ from tracings using some of our own high- $z$ galaxies. The difference between the two measurement methods turned out to be $\leq 10 \%$. Although the spectral resolutions of the different investigations are not exactly the same, the individual resolutions are sufficiently close to allow a direct comparison within the accuracy needed here. Table 4

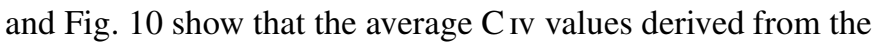
literature high- $z$ spectra are in reasonably good agreement with the mean values derived for the galaxies investigated in this work. However, the scatter of the literature data at high redshift is larger. Although this larger scatter is presumably dominated

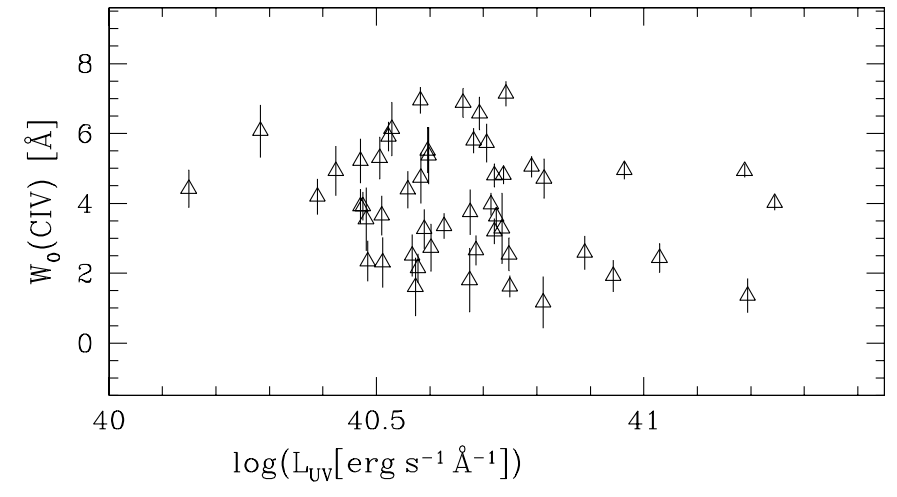

Fig. 9. Measured C Iv $\lambda 1550$ rest-frame equivalent widths of the high$z$ FDF galaxies as a function of the UV luminosity $L_{\mathrm{UV}}$, derived from the flux between $1432 \AA$ and $1532 \AA$ (as defined by Kinney et al. 1993).

by the on average low $\mathrm{S} / \mathrm{N}$ of the literature spectra, we cannot exclude that environmental effects may influence the evolution of the $\mathrm{C}_{\text {IV }}$ strength at high redshifts.

The best investigated individual high- $z$ galaxy is, so far, the gravitationally magnified object MS1512-cb58 $(z=2.727)$ (cf. e.g. Yee et al. 1996; Seitz et al. 1998; Pettini et al. 2000; Teplitz et al. 2001; Savaglio et al 2002). By measuring the C IV equivalent width on low resolution spectra and using Eq. (3) we obtain for this galaxy a metallicity of $0.4 Z_{\odot}$ and $0.2 Z_{\odot}$ from P00's and Y96's data, respectively. Within our error limits these values are in good agreement with the result of Pettini et al. (2000) (who derive $0.25 Z_{\odot}$ by comparing the galaxy spectrum with synthetic starburst galaxy spectra from Leitherer et al. 2001) and Teplitz et al. 2001 (who found $0.32 Z_{\odot}$ from measuring its oxygen abundance on NIR spectra using the strong line index $R_{23}$ which relates $(\mathrm{O} / \mathrm{H})$ to the relative abundance of [OII], [OIII] and $\mathrm{H} \beta$ ). This comparison seems to support our assumption that our calibration of the $\mathrm{C}_{\mathrm{IV}}$ strength in terms of metallicity is applicable to high redshift objects at $z=2.7$, although the redshift of MS1512-cb58 is too small to estimate the accuracy of the method for the interesting $z>3$ objects.

For four further high- $z$ Lyman-break galaxies Pettini et al. (2001) determined the oxygen abundance from NIR spectra using the strong line index $R_{23}$. Since the relation between $R_{23}$ and $\mathrm{O} / \mathrm{H}$ has an upper and a lower branch these abundances show the well-known two-value ambiguity. Hence these results do not provide a reliable test of our conclusions. Nevertheless, in Fig. 11 we plot the allowed ranges of oxygen abundance for these 4 Lyman-break galaxies as well as for MS 1512-cb58 together with the metallicities of our starburst galaxies (as derived from the $\mathrm{C}$ Iv strength via the calibration described above) as a function of redshift. This comparison shows that all data are at least mutually compatible, although for the two highest $z$ galaxies from Pettini et al. (2001) only the lower-branch results are in reasonable agreement with a strong increase of metallicity from redshift $\approx 3.2$ to $\approx 2.3$ suggested by our results.

Our results are also in line to those obtained by de Breuck et al. (2000), who find a qualitative increase of metallicity from higher to lower redshift for a sample of high- $z$ radio galaxies. Furthermore Pettini et al. (1997) and Savaglio et al. (2000) 


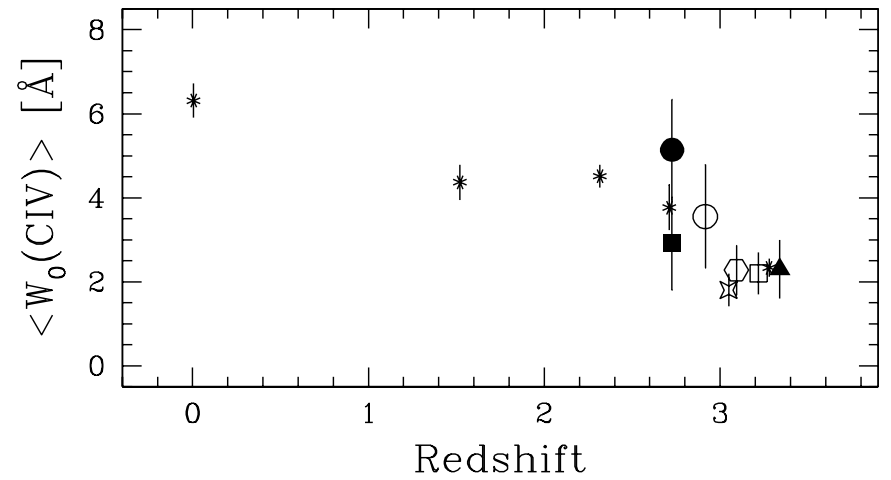

Fig. 10. Comparison of the observed $\mathrm{C}$ iv $\lambda 1550$ rest frame equivalent widths as a function of redshift for our galaxy sample (asterisks) with the mean values of $W_{0}(\mathrm{C}$ iv $)$ derived from high- $z$ galaxy spectra from Pettini et al. 1998 (P98; open circle), Steidel et al. (1996a) (S96; open square), Steidel et al. (1998) (S98; open hexagons) and Lowenthal et al. (L98; open diamond). The bars denote mean errors. Single measurements from galaxies observed by Pettini et al. (2000) (P00; filled circle), Yee et al. (1996) (Y96; filled square) and Trager et al. (1997) (T97; filled triangle) are also shown. Note that the Y96 and P00 observed the same object namely the well known lensed galaxy MS1512-cb58.

Table 4. Lines 1-4: Average of the measured C Iv $\lambda 1550$ rest-frame equivalent widths for high- $z$ galaxies observed by Pettini et al. (1998; P98), Lowenthal et al. (1997; L97) and Steidel et al. (1996a \& 1998; S96 \& S98). Lines 5-7: Measured C Iv $\lambda 1550$ rest frame equivalent width for MS1512-cb58 observed by Yee et al. (1996; Y96) and Pettini et al. (2000; P00) and for object DG-433 observed by Trager et al. (1997; T97). A * indicates that the equivalent widths were measured using the same method applied to the the high- $z$ galaxies presented in this work. $\mathrm{A} \dagger$ indicates that the equivalent widths were measured from enlarged tracings.

\begin{tabular}{ccccc}
\hline \hline$<z>$ & $\begin{array}{c}<\mathrm{C}_{\text {IV }}> \\
{[\AA]}\end{array}$ & $\begin{array}{c}\text { m.e.(C IV }) \\
{[\AA]}\end{array}$ & $N$ & Reference \\
\hline 2.92 & 3.55 & 1.23 & 5 & ${\mathrm{P} 98^{*}}^{\dagger}$ \\
3.05 & 1.80 & 0.39 & 5 & ${\mathrm{~L} 97^{\dagger}}^{\dagger}$ \\
3.09 & 2.28 & 0.59 & 4 & ${\mathrm{~S} 98^{\dagger}}^{\dagger}$ \\
3.22 & 2.20 & 0.50 & 2 & $\mathrm{~S} 96^{\dagger}$ \\
\hline$<z>$ & $W_{0}\left(\mathrm{C}_{\text {IV }}\right)$ & $\left.\mathrm{d} W_{0}\left(\mathrm{C}_{\text {IV }}\right)\right)$ & $N$ & Reference \\
& {$[\AA]$} & {$[\AA]$} & & \\
\hline 2.73 & 5.14 & 1.20 & 1 & $\mathrm{P}^{*}$ \\
2.73 & 2.91 & 1.11 & 1 & $\mathrm{Y}^{*}$ \\
3.34 & 2.30 & 0.70 & 1 & $\mathrm{~T}^{*}$ \\
\hline
\end{tabular}

report on evidence for a gradual chemical enrichment of the gas producing the damped Ly $\alpha$ lines in QSO spectra, although their trends are only weakly significant. Compared to Savaglio et al. (2000) we find a zero point offset of the metallicity-redshift relation of about 0.7 in $\Delta \log Z$ at $z=2.5$. Such a difference is not unexpected since the metal absorbers in damped Ly $\alpha$ systems most likely sample the outermost regions of galaxies and therefore a different environment than the dense interstellar matter of which the massive stars seen in starbursts have been formed.

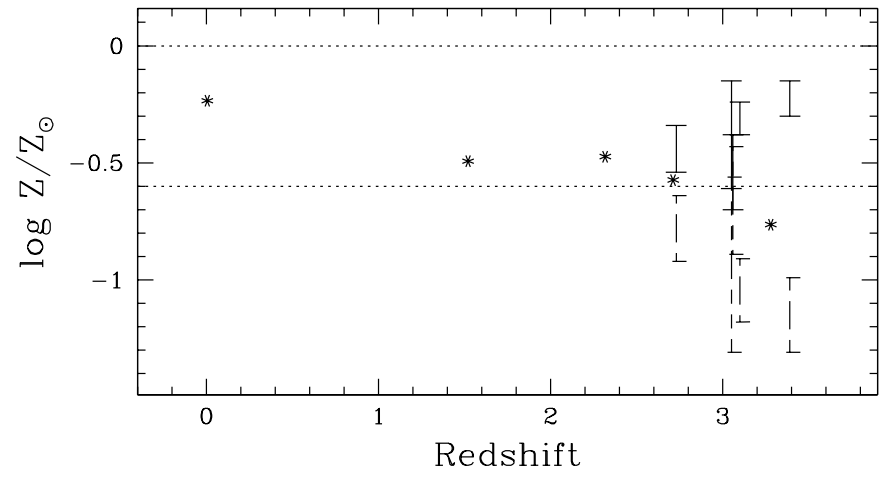

Fig. 11. Mean of the estimated metallicities as a function of redshift for all galaxies shown in Fig. 3. The oxygen abundances and the statistical uncertainties derived from $R_{23}$ ratio for 5 Lyman-break galaxies investigated by Pettini et al. (2001) and Teplitz et al. (2000) are indicated by the vertical lines: solid line: upper branch results; dashed line: lower branch result. Solar and LMC metallicities are indicated by the horizontal dotted lines.

\section{Conclusions}

Our study shows that the FDF starburst galaxies at $z \approx 3.2$ have on average significantly lower $\mathrm{C}$ IV equivalent widths than starburst galaxies at lower redshifts. In view of the known close relation of the C IV strength to the metallicity in local starburst galaxies, it appears likely that this effect is due to a significant evolution of the average metallicity in such objects at high redshifts. Using data from well studied local starburst galaxies we calibrated the C IV strength in terms of the heavy element content of these objects. Assuming that this calibration is applicable to high redshift starburst galaxies we find that the mean cosmic metallicity as observed in starburst galaxies has increased significantly between the cosmic epochs corresponding to $z \approx 3.2$ and $\approx 2.3$ (when the universe was between 2 and 3 Gyrs old). If this interpretation of the increase of the $\mathrm{C}$ IV absorption strength with decreasing redshift is correct, an intense phase of star formation and evolution of massive stars must have occurred during this period. At lower redshifts $(z<2.5)$ our data indicate little further increase of the average metallicity of starburst galaxies. Hence, the further cosmic chemical enrichment seems to have been insignificant during the last 11 Gyrs. The metallicity evolution indicated by our data is in reasonable agreement with published theoretical chemical enrichment models due to star formation at early cosmological epochs. Our results are also in agreement with a metallicity evolution found in high- $z$ radio galaxies (de Breuck et al. 2000) and the tentative evidence for a gradual chemical enrichment of the gas producing the damped Ly $\alpha$ lines (Pettini et al. 1997; Savaglio et al. 2000).

Since the FDF observations provide a fairly complete sample of the bright starburst galaxies in the observed direction and redshift ranges, the observed chemical evolution should be characteristic of the cosmic volumes with the most intense star formation at the corresponding epochs. However, it is also clear, that our results do not apply to all objects and volumes at a certain redshift or epoch. It is, e.g., well known that the BLR gas of high-redshift QSOs is characterized by high 
metallicities and that no significant chemical evolution is observable in these objects up to at least $z=5$ (see e.g. Hamann \& Ferland 1999; Dietrich et al. 1999; Dietrich \& WilhelmErkens 2000). Obviously, in the environment of these early QSOs much star formation and stellar evolution must have taken place at epochs corresponding to even higher redshifts. It is also known, however, (and confirmed from the FDF survey) that high- $z$ starburst galaxies are much more frequent than bright high- $z$ QSOs. Hence, the starburst galaxies are expected to be a more representative tracer of the history of the overall cosmic chemical evolution than the QSOs.

From our data we cannot determine whether at high redshift a metallicity-luminosity relation does exist, since we do not have any faint objects in our high- $z$ sample. But it is evident that the high-redshift galaxies are on average overluminous for their metallicities compared with local starburst galaxies. This trend is also found by Pettini et al. (2001) and Kobulnicky \& Koo (2000) for Lyman break galaxies. From tests on various subsamples we find that the observed dependence of $W_{0}(\mathrm{C}$ Iv $)$ on redshift is not caused by a luminosity effect. Furthermore we showed that this dependence is also not cause by the possible selection effect of preferentially observing galaxies with low dust content and hence low metallicity at high redshifts.

Differences in the Si IV to $\mathrm{C}$ IV ratios between local and high- $z$ galaxies in our sample suggest differences in the population and star formation history in the galaxies with $z>3$. Short bursts of star formation may have been more important (relative to periods of "continuous star formation") at these early epochs.

Acknowledgements. We are greatly indebted to Drs. M. Pettini and H. K. C. Yee for providing the ASCII files of their high redshift spectra published in Yee et al. (1996) and Pettini et al. (1998, 2000). We thank the referee C. Leitherer for valuable comments. We also want to thank C. Tapken for helpful comments and the Paranal staff for their support. This research was supported by the German Science Foundation (DFG) (Sonderforschungsbereiche 375 and 439).

\section{References}

Appenzeller, I., Bender, R., Böhm, A., et al. 2000, ESO Messenger, 100,44

Bender, R., Appenzeller, I., Böhm, A., et al. 2001, in the ESO/ECF/STScI Workshop on Deep Fields, ed. S. Cristiani, A. Renzini, \& R. Williams, 96

Cristiani, S., Appenzeller, I., Arnouts, S., et al. 2000, A\&A, 359, 489

De Breuck, C., Röttgering, H., Miley, G., et al. 2000, A\&A, 362, 519

Dietrich, M., \& Wilhelm-Erkens, U. 2000, A\&A, 354, 17

Dietrich, M., Appenzeller, I., \& Wagner, S. J. 1999, A\&A, 352, L1

Fritze-v. Alvensleben, U., Fricke, K. J., et al. 1989, A\&A, 224, L1

Gabasch, A., et al. 2002, in preparation

Hamann, F., \& Ferland, G. 1999, ARA\&A, 37, 487

Heckman, T. M., Robert, C., Leitherer, C., et al. 1998, ApJ, 503, 646

Heidt, J., Appenzeller, I., Gabasch, A., et al. 2002, A\&A, submitted
Heidt, J., Appenzeller, I. Bender, R., et al. 2001, Rev. Mod. Astron. 14, Dynamic Stability and Instabilities in the Universe, ed. R. E. Schielicke, 209

Kauffmann, G. 1996, MNRAS, 281, 487

Kinney, A. L., Bohlin, R. C., Calzetti, D., et al. 1993, ApJS, 86, 5

Kobulnicky, H. A., \& Koo, D. C. 2000, ApJ, 545, 712

Kobulnicky, H. A., \& Zaritsky, D. 1999, ApJ, 511, 188

Leitherer, C., Leao, J. R. S., Heckman, T. M., et al. 2001, ApJ, 550, 724

Leitherer, C., Robert, C., \& Heckman, T. M. 1995, ApJS, 99, 173

Lowenthal, J. D., Koo, D. C., Guzman, R., et al. 1997, ApJ, 481, 673 (L97)

Madau, P. 2000, Proc. of the ESO VLT Opening Symp. From Extrasolar Planets to Cosmology, ed. J. Bergeron, \& A. Renzini, 52

Madau, P., Ferguson, H. C., Dickinson, M. E., et al. 1996, MNRAS, 283, 1388

Mas-Hesse, J. M., \& Kunth, D. 1999, A\&AS, 88 , 399

Mehlert, D., Noll, S., Appenzeller, I., and the FDF team 2002, Proc. of MPA/ESO/MPE/USM Joint Astronomy Conf. on Lighthouses of the Universe, ed. R. Sunyaev, M. Gilfanov, \& E. Churazov, in press

Mehlert, D., Seitz, S., Saglia, R. P., et al. 2001, A\&A, 379, 96

Möller, C. S., Fritze-v.Alvensleben, U., \& Fricke, K. J. 2002, A\&A, submitted

Noll, S., Mehlert, D., Appenzeller, I., et al. 2002, in preparation

Pauldrach, A. W. A., Puls, J., Kudritzki, R.-P., \& FButler, K. 1990, A\&A, 228, 125

Pettini, M., Shapley, A. E., Steidel, C. C., et al. 2001, ApJ, 554, 981

Pettini, M., Steidel, C. C., Adelberger, K. L., et al. 2000, ApJ, 528, 96 (P00)

Pettini, M., Kellogg, M., Steidel, C. C., et al. 1998, ApJ, 508, 539 (P98)

Pettini, M., Smith, L. J., King, D. L., et al. 1997, ApJ, 486, 665

Renzini, A. 2000, Proc. of the ESO VLT Opening Symp. From Extrasolar Planets to Cosmology, ed. J. Bergeron, \& A. Renzini, 168

Renzini, A. 1998, ASP Conf. Ser., 146, 298

Savage, B. D., \& Massa, D. 1987, ApJ, 314, 380

Savaglio, S., Panagia, N., \& Padovani, P. 2002, ApJ, 567, 702

Savaglio, S., Panagia, N., \& Stiavelli, M. 2000, in Cosmic evolution and galaxy formation: Structure, Interactions, and Feedback, ed. J. Franco, E. Terlevich, O. Lopez-Cruz, \& I. Aretxaga, ASP Conf. Ser., 215, 65

Seitz, S., Saglia, R. P., Bender, R., et al. 1998, MNRAS, 298, 945

Steidel, C., Adelberger, K. L., Dickinson, M., et al. 1998, ApJ, 492, 428 (S98)

Steidel, C., Giavalisco, M., Pettini, M., et al. 1996a, ApJ, 462, L17 (S96)

Steidel, C., Giavalisco, M., Dickinson, M., et al. 1996b, AJ, 112, 352

Teplitz, H. I., McLean, I. S., Becklin, E. E., et al. 2000, ApJ, 533, L65

Trager, C., Faber, S. M., Dressler, A., et al. 1997, ApJ, 485, 92 (T97)

Walborn, N. R., Lennon, D. J., Haser, S. M., et al. 1995, PASP, 107, 104

Walborn, N. R., \& Panek, R. J. 1984, ApJL, 280, L27

Yee, H. K. C., Ellingson, E., Bechtold, J., Carlberg, R. G., \& Cuillandre, J.-C. 1996, AJ, 111, 1783 (Y96) 